\title{
3
}

\section{Motors in muscle: the function of conventional myosin II}

\author{
Clive R. Bagshaw \\ Department of Biochemistry, University of Leicester, Leicester \\ LE1 $7 R H, U . K$.
}

\section{Introduction}

The study of the mechanism of muscle contraction has a long history and the ideas that have emanated from this field have laid the foundation for much of what we know about molecular motors. Skeletal muscle, in particular, has been the target for investigation because its structural organization provides clues to the mechanism of contraction, and the abundance of contractile proteins within it allows ready isolation of its components for biochemical characterization $[1,2]$. In skeletal muscle, the key proteins, actin and myosin (of the conventional two-headed myosin-II class) are organized in linear arrays, making up the so-called thin and thick filaments of the sarcomere (Figure 1). A key observation in the 1950s was that, during contraction, these filamentous assemblies did not shorten but slid past one another.

Two decades earlier, myosin was shown to have ATPase activity; a crucial result at a time when ATP was identified as a central player in bioenergetics. However, demonstrating that ATP is the prime energy source for muscle contraction proved difficult, owing to the efficient regenerating systems in vivo. The advent of motility assays in vitro in the 1980s marked a climax in the biochemical approach to understanding the molecular basis of contraction; protein-filament sliding could be observed by eye in real time using an optical microscope. A minimal system comprising purified F-actin, the myosin head domain and MgATP was demonstrated to be sufficient for mechanochemical 
coupling. The intricate organization of skeletal muscle is an adaptation for achieving rapid macroscopic movement and to develop high tension, but the fundamental transduction event is a property of a unitary interaction between two proteins.

Over these last two decades there have been impressive advances in technology that have opened up the field of molecular motors beyond the specialized topic of muscle contraction. Use of recombinant proteins and sensitive functional assays has removed some of the early advantages of investigating muscle proteins. Nevertheless, muscle remains a particularly attractive system for investigation because the conclusions from the study of unitary events at the protein level can be critically evaluated at the macroscopic level. Only when agreement is reached can we claim to have a clear understanding of the processes involved.

\section{Identification of myosin crossbridges}

Following the demonstration that muscle shortening involved the interdigitation of thick and thin filaments, the problem to be solved was rephrased as 'what makes filaments slide?'. On the basis of a number of properties of intact muscle, including that the tension which developed was proportional to the degree of overlap of the filaments whereas shortening velocity was independent, A.F. Huxley proposed that sliding was driven by the repetitive action of individual 'side pieces' that transiently connected to two filament types. In the same year, H.E. Huxley showed, using electron microscopy, the existence of protrusions from the thick filament that could link to the actin filament. The concept of the myosin crossbridge was born (for reviews, see $[1,2])$.

Solubilization of the thick filament yielded myosin as the dominant component; a protein with two pear-shaped heads about $16 \mathrm{~nm}$ long attached to a 160-nm-long tail. At physiological ionic strength, the tails pack together to form the filament backbone, while the heads project to make up the crossbridges. The tail is known to be a coiled-coil $\alpha$-helix from characteristic X-ray fibre diffraction spots, whereas the head structure was solved by crystallography [3]. This form of myosin was termed myosin II to distinguish it from the single-headed myosin-I class and other members of the myosin superfamily identified subsequently. Structural analysis of the myosin-II molecule (termed simply myosin in the remainder of this chapter) benefited from partial proteolysis studies. Digestion with a number of proteases of different specificities showed accessible regions near the base of each head, yielding individual subfragment 1 (S1) moieties, and also about one-third of the way into the tail, yielding a two-headed fragment containing a short tail (heavy meromyosin, HMM). The remainder of the tail (light meromyosin) forms filamentous structures at physiological ionic strength, whereas the short tail attached to HMM (subfragment 2, S2) is soluble at low ionic strength. 


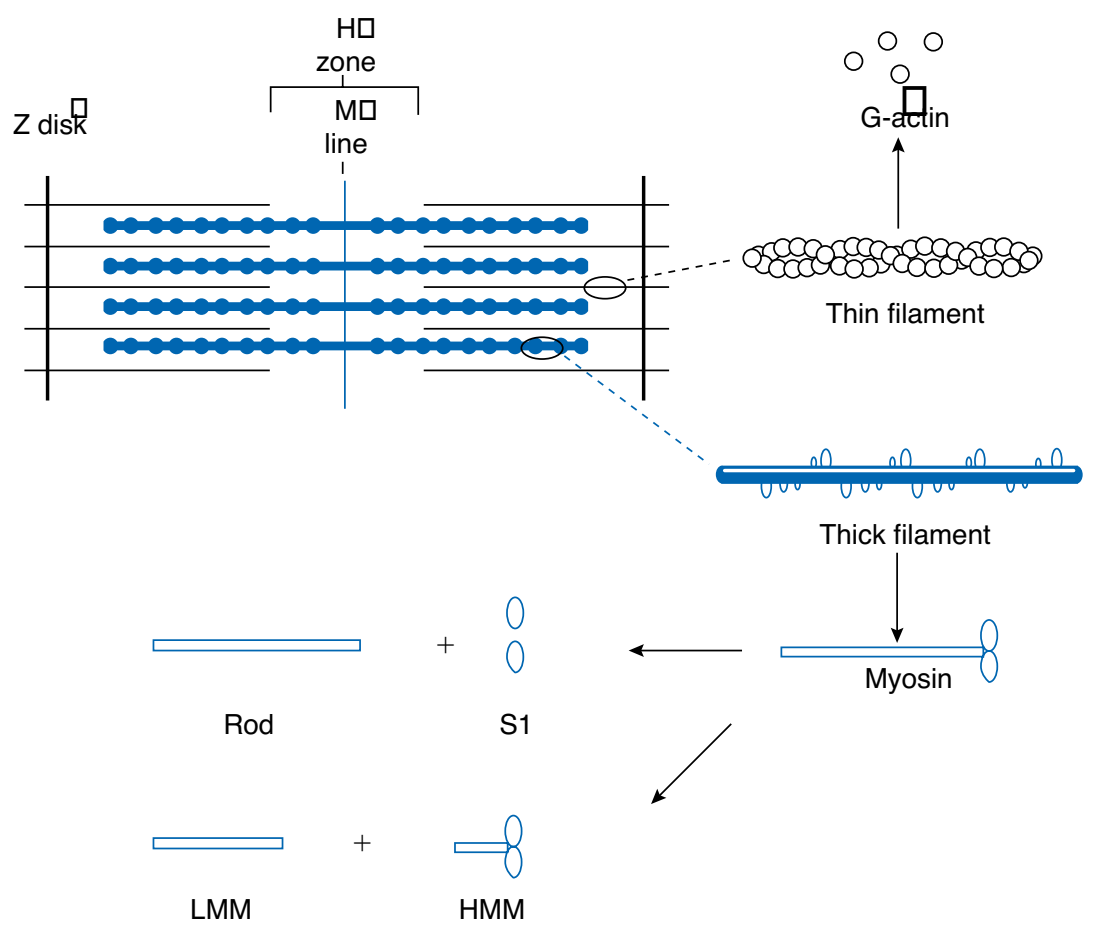

Figure 1.Schematic diagram of a sarcomere, the organelle of contraction in striated muscle

Disruption of the lattice allows the isolation of thick and thin filaments which can be solubilized to yield primarily myosin and actin, respectively. LMM, light meromyosin; HMM, heavy meromyosin.

The action of crossbridges has been studied from a number of different standpoints and using preparations ranging from intact muscle to isolated proteins. The regular arrangement of actin and myosin molecules within filaments and of the filaments within the muscle fibre has greatly aided the analysis by electron microscopy and X-ray diffraction. Early electron micrographs established that the myosin crossbridges attach to the actin filaments when the muscle fibre is depleted of ATP (i.e. the rigor state), in line with physiological measurements showing a very high stiffness. In the presence of ATP and the absence of $\mathrm{Ca}^{2+}$, the crossbridges are detached and the muscle is flaccid (i.e. the relaxed state). On stimulation of intact muscle, or addition of ATP plus $\mathrm{Ca}^{2+}$ in the case of permeabilized preparations, tension is developed if the muscle is prevented from shortening (i.e. the isometric state), but analysis of the structural state(s) of the crossbridge by electron microscopy is difficult because the fixation procedures are likely to perturb the distribution of dynamic states. More recently, rapid freezing followed by cryoelectron microscopy has been used in an attempt to overcome this problem [4].

$\mathrm{X}$-ray diffraction of intact muscle provides a complementary non-destructive approach. Several of the diffraction spots in the pattern can be assigned to 
particular spacings between protein units within the muscle and clear changes are seen on going from the relaxed to the contracted states and to the rigor state. The combined conclusions from these structural studies are that in rigor most, if not all, the myosin heads attach to the actin with a high degree of stereospecificity, with the main axis of the myosin head lying $\approx 45^{\circ}$ to the fibre axis. In relaxed muscle most of the heads lie close to the filament backbone, while in isometric contraction there is more disorder, indicating that a distribution of crossbridge states exists, although a significant proportion appears to be attached.

The angled attached crossbridge in the rigor state is thought to represent the end-state of a contraction cycle. If the crossbridge was to attach to the actin filament at an angle near to the normal and then tilt to the rigor position, a relative translational movement of the order of $10 \mathrm{~nm}$ would be achieved between the filaments. If the filament ends were fixed, then tension would develop from the resultant strain in the crossbridge: to what extent the crossbridge would move under such isometric conditions depends on the location of the compliance in the system. Direct evidence for this swinging crossbridge model has proven exceedingly difficult to acquire. One reason for this may be the fact that only a fraction of the heads are attached at any one instant and during rapid shortening this may be a very small fraction indeed. Furthermore, the heads act asynchronously. Thus any structural signal is present on a high background from crossbridges in other intermediate states.

\section{Myosin ATPase activity and actin activation}

Myosin from vertebrate skeletal muscle hydrolyses MgATP slowly $\left(k_{\text {cat }}=0.05 \mathrm{~s}^{-1}\right)$, but this activity is accelerated by two orders of magnitude by F-actin at low ionic strength. While such activation shows a roughly hyperbolic dependence on the actin concentration, interpretation of $V_{\max }$ and $K_{\mathrm{m}}$ is difficult because the actin and myosin sites are positioned on filamentous arrays that form a poorly organized meshwork, and hence the local concentrations and the availability of sites are unclear. For this reason most mechanistic studies have been performed using soluble myosin fragments (S1 and HMM). The simplest scheme that can describe the ATPase mechanism is given in eqn. (1) (where $\mathrm{M}$ is a myosin head):

$\mathrm{M}+\mathrm{ATP} \rightleftharpoons \mathrm{M} \cdot \mathrm{ATP}+\mathrm{H}_{2} \mathrm{O} \rightleftharpoons \mathrm{M} \cdot \mathrm{ADP} \cdot \mathrm{P}_{\mathrm{i}} \rightleftharpoons \mathrm{M}+\mathrm{ADP}+\mathrm{P}_{\mathrm{i}}$

Although the steady-state ATPase activity of myosin is slow, the hydrolysis step itself is rapid (about $100 \mathrm{~s}^{-1}$ ) and leads to a long-lived products complex $\left(\mathrm{M} \cdot \mathrm{ADP} \cdot \mathrm{P}_{\mathrm{i}}\right)$ as the major steady-state intermediate. This was established using quenched-flow techniques to acid-denature the myosin after mixing with excess ATP on a millisecond time scale. A burst of $P_{i}$ and ADP production was observed, corresponding to the release of products from denatured $\mathrm{M} \cdot \mathrm{ADP} \cdot \mathrm{P}_{\mathrm{i}}$ formed during the first turnover, whereas quenching at longer 
times revealed additional products produced at the slow steady-state rate. The hydrolysis step is readily reversible and in rapid equilibrium so that a significant concentration (typically 10-20\%) of the nucleotide is present as the $\mathrm{M} \cdot \mathrm{ATP}$ complex. Product release is limited by an isomerization step after which $P_{i}$ is rapidly released and ADP somewhat slower. Intrinsic fluorescence and extrinsic probes demonstrate that the conformations of the M, M.ADP and $\mathrm{M} \cdot \mathrm{ADP} \cdot \mathrm{P}_{\mathrm{i}}$ states differ. The M.ATP state is more difficult to characterize but may share some similarities with the $\mathrm{M} \cdot \mathrm{ADP}$ state.

During the steady-state turnover of ATP by a myosin head, the predominant intermediate is $\mathrm{M} \cdot \mathrm{ADP} \cdot \mathrm{P}_{\mathrm{i}}$ but with significant contributions from $\mathrm{M} \cdot \mathrm{ATP}$ and M.ADP binary complexes, particularly at low temperatures. This mixture complicates the definition of structural studies and also, after several minutes, the ATP becomes exhausted, limiting the time for data capture. To overcome this problem analogue states have been developed. Simply adding high concentrations of $\mathrm{P}_{\mathrm{i}}$ to $\mathrm{M} \cdot \mathrm{ADP}$ does not generate significant amounts of $\mathrm{M} \cdot \mathrm{ADP} \cdot \mathrm{P}_{\mathrm{i}}$; indeed, $\mathrm{P}_{\mathrm{i}}$ tends to displace the ADP and forms a $\mathrm{M} \cdot \mathrm{P}_{\mathrm{i}}$ complex. However, it turns out that a number of compounds with a similar size and shape to $\mathrm{P}_{\mathrm{i}}$ will form a $\mathrm{M} \cdot \mathrm{ADP} \cdot \mathrm{P}_{\mathrm{i}}$-like state, notably $\mathrm{VO}_{4}{ }^{3-}, \mathrm{AlF}_{4}{ }^{-}$and $\mathrm{BeF}_{3}{ }^{-}$. A characteristic of these complexes is that they form slowly (a time scale in the order of minutes) but dissociate over several hours or days, giving ample time for structural measurements.

In the absence of nucleotide, S1 binds tightly to F-actin $\left(K_{\mathrm{d}}=10-100 \mathrm{nM}\right)$ to give decorated filaments in which the $\mathrm{S} 1$ heads project at the characteristic rigor angle. Addition of ATP causes dissociation of the S1 heads but, somewhat paradoxically, the S1 ATPase activity is enhanced. The solution to this paradox came with Lymn \& Taylor's [5] investigations comparing the actoHMM dissociation rate constant, measured by using turbidity in a stoppedflow apparatus, with the hydrolysis rate constant measured by the quenchedflow technique. They concluded that the A·M·ATP complex (where A is actin) rapidly dissociates and that hydrolysis occurs predominantly on the dissociated myosin (i.e. the same M.ATP to M.ADP. $P_{i}$ transition as in eqn. 1). In order to explain the activation of the ATPase, it was proposed that actin rebound to the $\mathrm{M} \cdot \mathrm{ADP} \cdot \mathrm{P}_{\mathrm{i}}$ state to bypass the slow myosin pathway (eqn. 2).

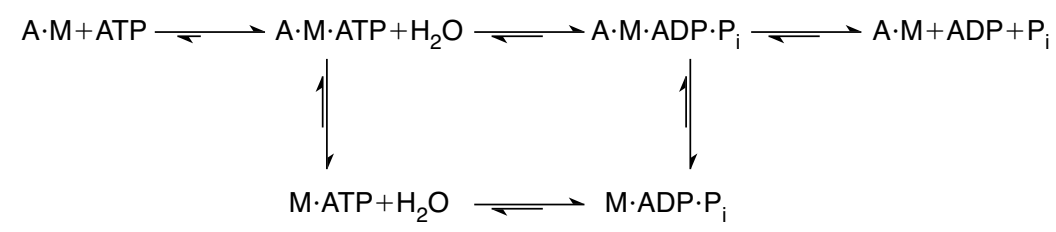

The dissociation and reassociation of the myosin head with actin during ATPase activity suggested a possible crossbridge cycle (Figure 2).

The Lymn-Taylor scheme remains the framework upon which many new results are discussed. However, it is an incomplete description of events. In 

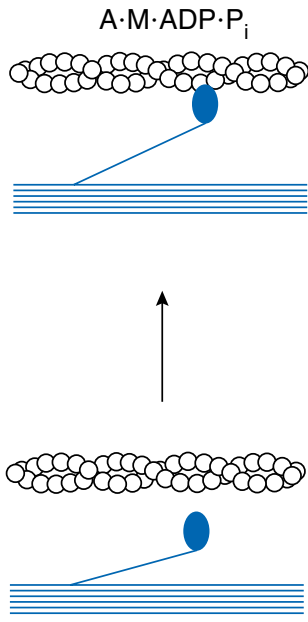

$M \cdot A D P \cdot P_{i}$
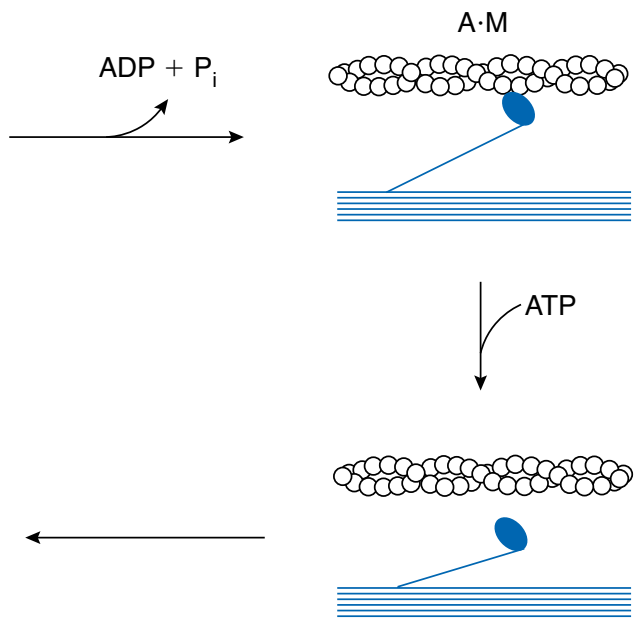

$M \cdot A T P$

Figure 2. The Lymn-Taylor scheme for mechanochemical coupling [5]

ATP dissociates the rigor complex (A.M). Hydrolysis of bound ATP occurs primarily on the detached head $\left(M \cdot A T P \leftrightarrow M \cdot A D P \cdot P_{j}\right)$, whereas $A D P$ and $P_{i}$ release is induced by actin rebinding. The scheme proposed originally suggested that the crossbridge stroke arises from tilting of the attached head, whereas recent data suggest it may bend to effect a similar translation of about $10 \mathrm{~nm}$ for each cycle.

their original work, Lymn \& Taylor [5] concluded that at high actin concentrations release of $\mathrm{P}_{\mathrm{i}}$ from the $\mathrm{A} \cdot \mathrm{M} \cdot \mathrm{ADP} \cdot \mathrm{P}_{\mathrm{i}}$ complex was the major rate-limiting step. Subsequently it was shown under some conditions that hydrolysis may become rate limiting, although in myofibrils held under isometric conditions, the overall turnover rate is slower than with isolated proteins and is limited by $\mathrm{P}_{\mathrm{i}}$ release [6]. This link between structure and chemistry is crucial and follows from thermodynamics: if the free energy of ATP hydrolysis is converted to mechanical work, then imposing a load on a muscle should affect the equilibrium, and hence kinetics, of the ATPase reaction.

These schemes (eqns. 1 and 2) have been expanded to incorporate changes in myosin conformation revealed by transient-state kinetics. In an attempt to simplify the relation between structure and nucleotide state, Shriver [7] analysed models in which there were two fundamental conformations of the myosin, and the equilibrium between the two was shifted by nucleotide binding. This concept was supported by spectroscopic evidence and seems to be in accord with recent X-ray crystallographic results (see below). However, Shriver [7] concluded that the degree to which the conformational state could be switched by nucleotide binding was insufficient to account for the observed changes in actin affinities in a thermodynamically self-consistent way, i.e. there are further conformational transitions that modulate actin affinity.

One of the crucial tests of the Lymn-Taylor model (Figure 2) is to gain structural information about the A.M.ADP. $\mathrm{P}_{\mathrm{i}}$ state prior to the putative crossbridge swing. Unfortunately analogues that favour such a state tend to result in 
(a)

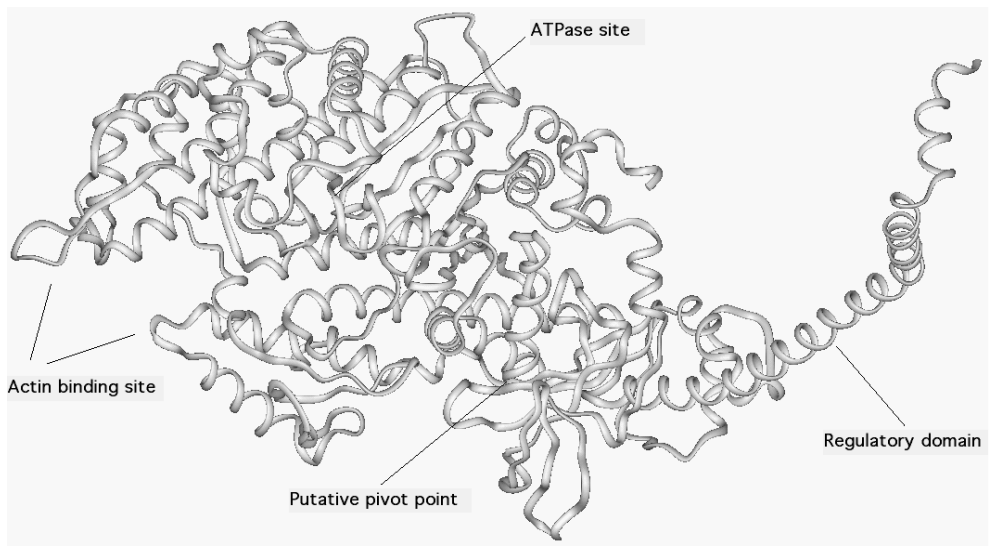

(b)

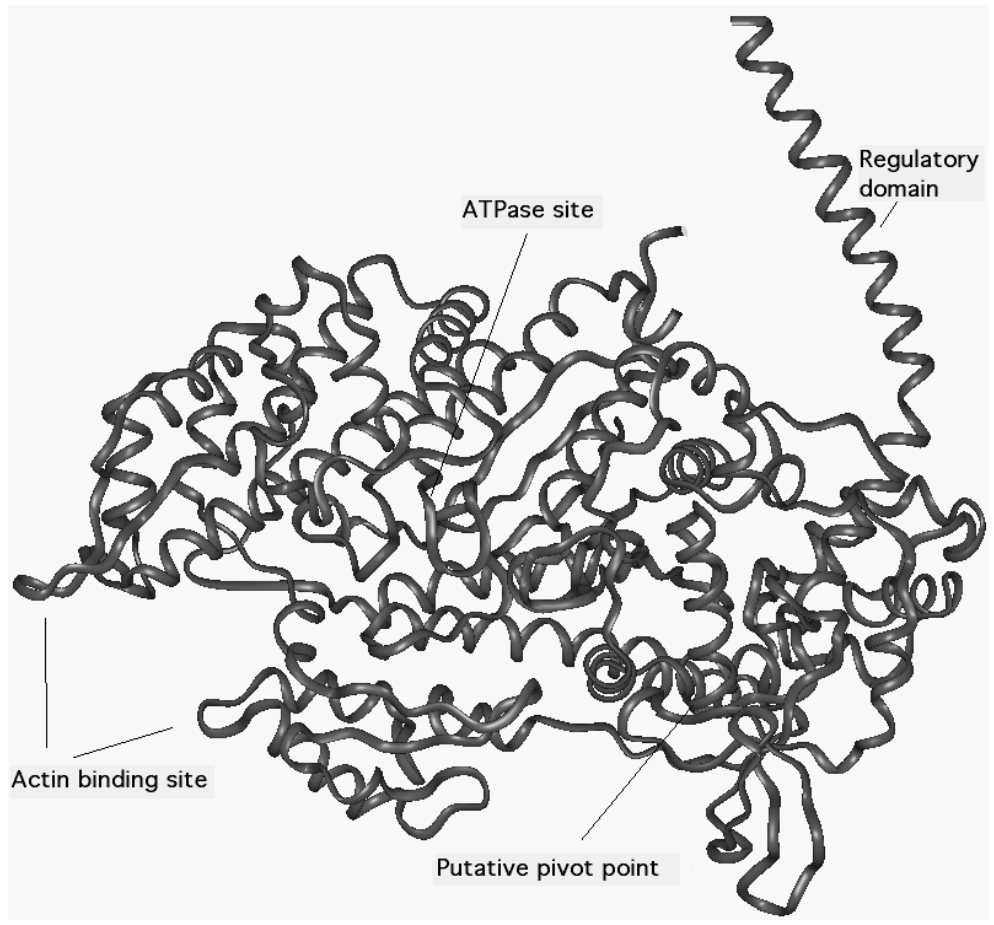

Figure 3. Comparison of the structures of skeletal-muscle $S I$ in its nucleotide-free

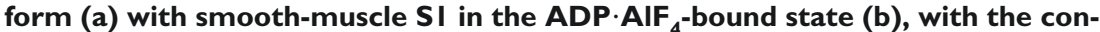
served $\beta$-sheet at the active site co-aligned to reveal about a $70^{\circ}$ tilt of the regulatory domain

The light chains have been left out for clarity. Data are from Brookhaven files 2MYS and IBRI and $[3,10]$.

actin dissociation. Working at very low ionic strength helps to counteract this, but significant dissociation may still occur. On the other hand, ADP is less 
efficient at dissociating actin and, under appropriate conditions, a significant concentration of the ternary $\mathrm{A} \cdot \mathrm{M} \cdot \mathrm{ADP}$ state can be generated, particularly with smooth-muscle proteins.

\section{X-ray crystallographic studies}

Crystallographic studies of actin and myosin present a challenge because both proteins spontaneously polymerize. In the case of actin, the crystal structure of the $G$ form was solved in combination with other proteins that keep it in the monomeric form (e.g. DNase, profilin, gelsolin). The structure of F-actin was then modelled by fitting the high resolution structure of $\mathrm{G}$-actin into the electron density envelope observed by electron microscopy [8].

Fragments of myosin, generated by proteolysis or recombinant DNA technology, have proven amenable to crystallization. To date, the key structures solved are chicken skeletal S1 (Figure 3a), containing both light chains and a sulphate ion (but no nucleotide) at the active site [3], a number of truncated Dictyostelium S1 constructs containing various nucleotides and analogues at the active site but lacking light chains [9], and a recombinant chicken smooth-muscle S1 containing an essential light chain and bound nucleotide [10]. Overall, the folds of the motor domain are very similar, and indeed the active site shares conserved motifs with kinesins and G-proteins [11]. In particular, the nucleotide-binding site contains three loops (the P-loop, switch 1 and switch 2), which form specific interactions with the triphosphate moiety.

Interestingly, structures of the Dictyostelium constructs with a variety of ligands fall into two classes that differ in the position of the switch-2 loop. Nucleotide-free states and analogues thought to mimic the M-ATP state $(\mathrm{M} \cdot \mathrm{ADP}$ and $\mathrm{M} \cdot \mathrm{ATP} \gamma \mathrm{S})$ resemble the original skeletal S1 structure. However, with analogues thought to mimic a transition state approaching the M.ADP. $P_{i}$ intermediate (ADP plus $\mathrm{AlF}_{4}{ }^{-}$or $\mathrm{VO}_{4}{ }^{3-}$ ), the switch-2 loop moves closer to the putative position of the $\gamma$-phosphate. This movement is coupled to the closure of the cleft between the upper and lower $50 \mathrm{kDa}$ domains, and a rearrangement of the $\mathrm{C}$-terminus. The latter suggested that the regulatory domain, missing in these structures, would project at a very different angle, but such speculation was tempered by the possibility that truncation induced an artefactual rearrangement of the C-terminus.

Fortunately this situation was clarified with the solution of the smoothmuscle $\mathrm{S} 1 \cdot \mathrm{ADP} \cdot \mathrm{AlF}_{4}{ }^{-}$structure (Figure $3 \mathrm{~b}$ ), where the regulatory domain was indeed rotated about $70^{\circ}$ relative to its position in the nucleotide-free skeletal $\mathrm{S} 1$ [10]. This movement, which pivots about a point in the $20 \mathrm{kDa}$ domain, corresponds to about a $10 \mathrm{~nm}$ displacement at the distal end of the regulatory domain, where it connects to $\mathrm{S} 2$ in the intact myosin molecule. Thus the regulatory domain, aside from acting as a control element in some myosins, may function as a lever arm. However, this study also revealed a complication in that the $\mathrm{M} \cdot \mathrm{ADP} \cdot \mathrm{BeF}_{3}$ structure with smooth-muscle $\mathrm{S} 1$ also took on the bent 
structure, whereas with the Dictyostelium construct, this analogue resembled the extended skeletal myosin structure. Perhaps this result is not unexpected in view of earlier solution studies. The M.ATP and M.ADP. $\mathrm{P}_{i}$ states differ very little in energy so that crystal forces could easily select one form over the other. Furthermore, reference was made above to the fact that any single nucleotide state may exist in two or more conformations in solution [7], which would be free to re-equilibrate as crystallization proceeded.

The location of the actin-myosin binding interface has been defined by fitting the crystal structures of G-actin and the myosin head into the envelope of density defined by electron microscopy of S1-decorated actin filaments. The overall fit suggests the head must be in the extended conformation close to that of skeletal S1 in the absence of bound nucleotide. Whereas the residues that are located at the interface can be identified from the fitting procedure, the precise contacts cannot be identified. The actomyosin interface is clearly multivalent, with electrostatic interactions predominately involved in a weak, non-stereo specific interaction, whereas hydrophobic interactions may drive the rearrangement to a more stable attachment. However, this interface must change in a dramatic way (in energetic terms) when nucleotide is bound to the active site, even if the structural reorganization is rather small. Communication between the nucleotide and actin sites may occur via the opening and closing of a cleft that runs between the so-called $50 \mathrm{kDa}$ domain. Movement of the switch-2 loop in the M.ADP. $\mathrm{AlF}_{4}$ structure is coupled to the closing of the cleft, and could account for its weak actin-binding properties. However, the more open state of the cleft observed with other nucleotides (e.g. ATP $\gamma$ S) is comparable in gross structure with that of the nucleotide-free state, yet the binding affinities for actin differ by several orders of magnitude.

Overall, crystallographic studies support the idea that the myosin head can bend, and if such a movement is reversed when it is attached to actin, it would account for a stroke size of about $10 \mathrm{~nm}$ in the correct direction. Furthermore, only about $35 \%$ of the myosin mass moves in this transition, which may account for the difficulty of resolving this process by fibre diffraction from whole muscle. However, our understanding of the effects of actin is incomplete.

\section{Evidence for lever-arm movement in solution studies}

The characterization of the lever-arm positions in the crystal structures begs the questions: 'does such a change occur in solution?' or, conversely, 'is the change an artifact of crystallization?' and 'does it occur when the myosin head is attached to actin?'. In fact, the question of whether the myosin head bends was asked long before the recent crystallographic results.

Solution measurements on isolated S1 heads using electric birefringence [12] and X-ray scattering [13] indicated a shift in conformation to a more compact form in the presence of bound nucleotide. Electron microscopy has also 
revealed that $\mathrm{S} 1$ heads can take on straight or bent conformations, although the nature of the coupling to the nucleotide state is controversial (compare [14] and [15]). Recently, a fusion protein has been made in which green fluorescent protein (GFP) was attached to the $\mathrm{N}$-terminus and blue fluorescent protein (BFP) was attached to the $\mathrm{C}$-terminus of the motor domain in place of the regulatory domain (Figure 4). This construct displays fluorescence resonance energy transfer (FRET) in which excitation of BFP results in dipolar coupling with GFP and emission of fluorescence characteristic of the latter, a phenomenon that is distance- and orientation-dependent. During ATP turnover the FRET efficiency between BFP and GFP changes, indicating a movement or reorientation of the $\mathrm{C}$-terminal region relative to the $\mathrm{N}$-terminus [16]. Interestingly, $\mathrm{BeF}_{3}{ }^{-}$(which may promote either of the two crystal conformations) gave FRET efficiencies halfway between those of the M and M-ADP.P states. The GFP approach opens the way to examining such conformational changes in the presence of actin.

Cross-linking studies between thiol residues on a helix at one end of the putative pivot point (see Figure 3) indicate a variable distance between the cysteine residues dependent on the state of the bound nucleotide $[17,18]$. The crystal structures described above both have an intact helix in which the thiols are about $1.8 \mathrm{~nm}$ apart. However, most recently, a third conformation has been solved by crystallography in which the helix is melted and the regulatory domain straightened compared with the original chicken structure [19].

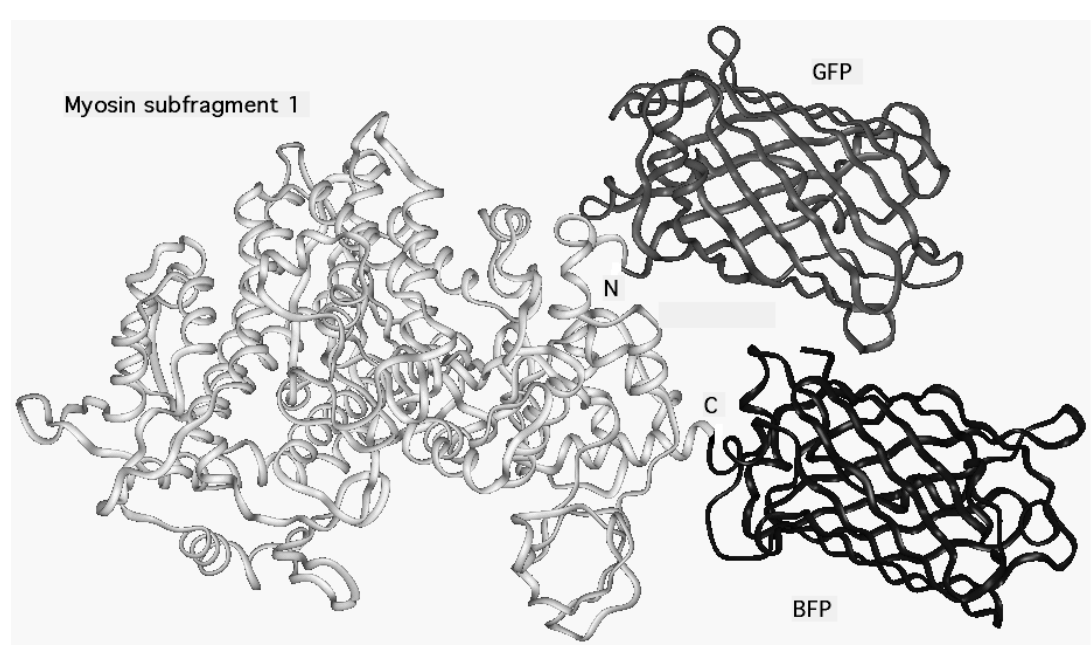

Figure 4. The use of GFP-BFP FRET pairs to follow conformational changes in the neck region of the myosin molecule [16]

ATP binding induces an isomerization that leads to a reorientation between the $\mathrm{N}$ - and $\mathrm{C}$ termini and relative movement of the GFP and BFP fluorophores (located within their $\beta$-cage structure). The transition is reversed on $\mathrm{P}_{\mathrm{i}}$ release [16]. The diagram was constructed using co-ordinates from Brookhaven files 2 MYS and IEMB. 
Evidence that the regulatory domain acts as a lever has come from motility assays in vitro using constructs that have artificially lengthened or shortened necks. In general there is a good correlation between the length of the lever arm and the observed sliding velocity [20]. Myosins in different classes show a wide range of lever-arm lengths, as judged by the number of light-chain-binding sites (one to six) and these may be adaptations to provide variable gearing to the task at hand.

Examination of head movements while attached to actin is more difficult because only a small fraction of the heads may be involved. In solution, high actin concentrations are required to favour binding, so in many cases it is more convenient to use permeabilized muscle fibres, which come with the added advantage of a high degree of alignment. As a test of the Lymn-Taylor model, many early studies attempted to monitor angular changes in the myosin head by attaching optical or magnetic probes to the motor domain, via such groups as the reactive thiols. In general, little evidence was produced for head attachment at a unique angle which differed from that of rigor, although it was considered that heads may attach over a range of angles, based on estimates of the fraction of heads attached. More recently, probes have been introduced via the light chains to test for a swing of the lever arm [21]. Small fluorescence polarization signals have been recorded from a rhodamine probe on the regulatory light chain that may be explained by a few heads (10-15\%) undergoing a large angle change $\left(>30^{\circ}\right)$. However, correlation with chemical states is difficult because different heads in the myofibril array experience different mechanical states.

\section{Problems and prospects}

The fundamental concepts of the swinging crossbridge model (i.e. physical attachment of the myosin head to actin followed by some form of structural rearrangement) have been with us for 40 years. These basic tenets have been challenged by alternative hypotheses, including ones that place the contractile event in myosin S2 or, more radically, have no direct physical link between actin and myosin and rely on indirect effects of long-range charges or water movements within the myofibril lattice. The recent demonstration of force generation by a single myosin head lacking S2 and an organized myosin lattice, and the direct observation of the coupling between head attachment to actin and a single ATP-turnover cycle, lend powerful support to the classical crossbridge theory [22]. These ideas are also supported by the discovery of the myosin superfamily of proteins, which retain a conserved motor domain, but are otherwise very diverse, and in some cases function as individual porters rather than in a filamentous array. Crystallography is now beginning to define the likely movements that the myosin head can make. The communication path between events at the active site and both the proximal actin-binding site and the distal regulatory domain are now being defined at the atomic level. 
Site-directed mutagenesis studies are providing critical tests for these ideas. However, these studies are still incomplete. In particular, events at the actin interface and conformational changes within actin itself are unclear.

Whereas there is now direct evidence that the turnover of a single ATP molecule by a singe $\mathrm{S} 1$ head can generate a translational movement $(5-15 \mathrm{~nm})$ comparable with that predicted by the swing or bend of the head itself, there remain some unexplained discrepancies. In particular, under conditions of low load, the overall ATPase rate and the working stroke size argue for a very low duty ratio if the mechanics and ATPase are coupled in a 1:1 fashion [1,2]. During sliding at maximum velocities, only about $1 \%$ of the heads can undergo this stroke at a particular instant. It is possible that some heads may attach and use their binding energy to impart an impulse to the actin, only to be ripped off by other heads before completing their ATPase cycle. Thus the number of attached heads may be higher than $1 \%$. By 'living on borrowed energy' the free energy of ATP hydrolysis may be partitioned over several heads [2]. Evidence for multiple strokes has been obtained within muscle fibres by imposing a rapid staircase of releases. Nevertheless, such a mechanism does not account for the results from some motility assays in vitro where the total number of available actin and myosin sites are limited [23].

\section{Summary}

- Solution measurements indicate that actin and myosin alternately bind and dissociate during one ATP bydrolysis cycle.

- Crystallographic studies indicate at least two basic conformations of the myosin bead exist in which the regulatory domain swings through an angle of about $70^{\circ}$.

- Actin must further modulate these conformations, but high-resolution information about the actomyosin interface is lacking.

- One-to-one coupling between the ATPase cycle and a mechanical cycle involving myosin-bead bending could account for about a $10 \mathrm{~nm}$ stroke size.

- At high sliding velocities, discrepancies remain which suggest that a myosin head may undergo repetitive interactions with actin for each ATP hydrolysed.

I am grateful to Dr. M.J. Sutcliffe for assistance with molecular graphics and Dr. P.B. Conibear for discussions. Financial support was provided by The Royal Society and the Wellcome Trust.

\section{References}

I. Bagshaw, C.R. (1993) Muscle Contraction, 2nd edn, Chapman and Hall, London

2. Cooke, R. (I997) Actomyosin interaction in striated muscle. Physiol. Rev. 77, 67I-697 
3. Rayment, I., Rypniewski, W.R., Schmidt-Bäse, K., Smith, R., Tomchick, D.R., Benning, M.M., Winkelmann, D.A., Wesenberg, G. \& Holden, H.M. (1993) Three-dimensional structure of myosin subfragment-I: a molecular motor. Science 26I, 50-58

4. Hirose, K., Lenart, T.D., Murray, J.M., Franzini-Armstrong, C. \& Goldman, Y.E. (1993) Flash and smash: rapid freezing of muscle fibers activated by photolysis of caged ATP. Biophys. J. $\mathbf{6 5}$, 397-408

5. Lymn, R.W. \& Taylor, E.W. (I97I) Mechanism of adenosine triphosphate hydrolysis by actomyosin. Biochemistry I0, 46I7-4624

6. Lionne, C., Brune, M., Webb, M.R., Travers, F. \& Barman, T. (1995) Time resolved measurements show that phosphate release is the rate limiting step on myofibrillar ATPases. FEBS Lett. 364, 59-62

7. Shriver, J.W. (1986) The structure of myosin and its role in energy transduction in muscle. Biochem. Cell. Biol. 64, 265-276

8. Holmes, K.C., Popp, D., Gebhard, W. \& Kabsch, W. (1990) Atomic model of the actin filament. Nature (London) 347, 37-44

9. Gulick, A.M. \& Rayment, I. (1997) Structural studies on myosin II: communication between distant protein domains. BioEssays 19, 56I-569

10. Dominguez, R., Freyzon, Y., Trybus, K.M. \& Cohen, C. (1998) Crystal structure of a vertebrate smooth muscle myosin motor domain and its complex with the essential light chain: visualization of the pre-power stroke state. Cell 94, 559-57I

II. Kull, F.J., Vale, R.D. \& Fletterick, R.J. (1998) The case for a common ancestor: kinesin and myosin motor proeins and G-proteins. J. Muscle Res. Cell Motility 19, 877-886

12. Highsmith, S. \& Eden, D. (1993) Myosin-ATP chemomechanics. Biochemistry 32, 2455-2458

13. Wakabayashi, K., Tokunaga, M., Kohno, I., Sugimoto, Y., Hamanaka, T., Takezawa, Y., Wakabayashi, T. \& Amemiya, Y. (1992) Small-angle synchrotron X-ray scattering reveals distinct shape changes of the myosin head during hydrolysis of ATP. Science 258, 443-447

14. Burgess, S.A., Walker. M.L., White, H.D. \& Trinick, J. (1997) Flexibility within myosin heads revealed by negative stain and single-particle analysis. J. Cell. Biol. I39, 675-68I

15. Bagshaw C.R. (1997) Myosin trapped but not tamed. Nature (London) 390, 345-346

16. Suzuki, Y., Yasunaga, T., Ohkura, R., Wakabayashi, T. \& Sutoh, K. (1998) Swing of the lever arm of the myosin motor at the isomerization and phosphate-release steps. Nature (London) 396, 380-383

17. Nitao, L.K. \& Reisler, E. (1998) Probing the conformational states of the SHI-SH2 helix in myosin: a cross-linking approach. Biochemistry 37, 16704-16710

18. Liang, W. \& Spudich, J.A. (1998) Nucleotide-dependent conformational change near the fulcrum region in Dictyostelium myosin II. Proc. Natl. Acad. Sci. U.S.A. 95, I 2844-I 2847

19. Houdusse, A., Kalabokis, V.N., Himmel, D., Szent-Györgyi, A.G. \& Cohen, C. (1999) Atomic structure of scallop myosin subfragment SI complexed with MgADP: a novel conformation of the myosin head. Cell 97, 459-470

20. Uyeda, T.Q.P., Abrahamson, P.D. \& Spudich, J.A. (1996) The neck region of the mysoin motor domain acts as a lever arm to generate movement. Proc. Natl. Acad. Sci. U.S.A. 93, 4459-4464

21. Goldman, Y.E. (1998) Wag the tail: structural dynamics of actomyosin. Cell 93, I-4

22. Ishijima, A., Kojima, H., Funatsu, T., Tokunaga, M., Higuchi, H., Tanaka, H. \& Yanagida, T. (1998) Simulatneous observation of individual ATPase and mechanical events by a single myosin molecule during interaction with actin. Cell 92, I6I-I7I

23. Saito. K., Aoki, T., Aoki, T. \& Yanagida, T. (1994) Movement of single myosin filaments and myosin step size on an actin filament suspended in solution by a laser trap. Biophys. J. 66, 769-777 